\title{
EMPERADOR INIA: NUEVA VARIEDAD DE TRITICALE (x Triticosecale Wittmack) DE INVIERNO PARA EL SUR DE CHILE
}

\author{
EMPERADOR INIA: A NEW WINTER TRITICALE (x Triticosecale Wittmack) \\ CULTIVAR FOR SOURTHERN CHILE
}

\author{
Claudio Jobet ${ }^{1}$, Javier Zúñiga ${ }^{1}$, Dalma Castillo ${ }^{2}$, Iván Matus ${ }^{2}$, Cristian Alfaro ${ }^{1}$, y Juan Carlos García ${ }^{3}$ \\ ${ }^{1}$ Instituto de Investigaciones Agropecuarias, Centro Regional Carillanca, Casilla 58-D, Temuco, Chile. \\ ${ }^{2}$ Instituto de Investigaciones Agropecuarias, Centro Regional Quilamapu, Casilla 426, Chillán, Chile. \\ ${ }^{3}$ Universidad de la Frontera, Facultad de Agronomía, Casilla 54-D, Temuco, Chile. \\ * Autor para correspondencia E-mail: cjobet@inia.cl
}

\section{RESUMEN}

Emperador INIA es una variedad de triticale ( $\times$ Triticosecale Wittmack) de invierno introducida por el Programa Nacional de Trigo del Instituto de Investigaciones Agropecuarias (INIA), Centro Rergional Carillanca, Chile, desde Nordsaat Saatzucht $\mathrm{GmbH}$, Alemania. Tiene hábito de crecimiento invernal, de porte muy rastrero al estado de inicio de macolla y sus hojas son de color verde pálido. El período vegetativo desde siembra a espigadura, sembrado en mayo, es de aproximadamente 180 días. Presenta moderada susceptibilidad al polvillo estriado (Puccinia striiformis West. f. sp. tritici Erikss.), resistencia al polvillo de la hoja (Puccinia triticina f. sp. tritici Erikss. y Henn) y oidio (Blumeria graminis D.C. f. sp. tritici Marchal), y moderada resistencia a septoriosis (Mycosphaerella graminicola (Fuckel) Sand). La altura de planta promedio es de $115 \mathrm{~cm}$, con tallo de muy buena resistencia a la tendedura. La espiga es de color rojo, compacta, y con barbas en toda su extensión. El grano es de forma alargada y de color rojo pálido. Tiene alto rendimiento, superior al mejor testigo en los ensayos comparativos de rendimiento, y buenas características de calidad para alimentación animal.

Palabras clave: cultivar, triticale, invierno, rendimiento, forraje

\section{ABSTRACT}

Emperador INIA is a winter triticale ( $\times$ Triticosecale Wittmack) variety introduced from Nordsaat Saatzucht GmbH of Germany by the National Wheat Breeding Program at INIA Carillanca, Chile. It has a postrate growth habit during the tiller stage, and its leaves are of pale green color. The vegetative period from sowing to flowering sown in May, is approximately 180 days. It has moderate susceptibility to stripe rust (Puccinia striiformis West. f. sp. tritici Erikss.), resistance to leaf rust (Puccinia triticina. f. sp tritici Erikss. y Henn), and powdery mildew (Blumeria graminis D.C. f.sp. tritici Marchal), and moderate resistance to septoria (Mycosphaerella graminicola (Fuckel) Sand). The average plant height is $115 \mathrm{~cm}$, with a stem of very good resistance to lodging. The spike is red, compact and with awns along its full length. The grain shape is elongated with a red pale color. It has high yield, higher than the best check in comparative yield trials, and good quality characteristics for animal feed.

Key words: cultivar, winter, triticale, yield, forages.

Recibido: 19 octubre 2018. Aceptado: 18 abril 2019. 


\section{INTRODUCCIÓN}

Las investigaciones relacionadas con el triticale ( $\times$ Triticosecale Wittmack), cereal que es producto de un cruzamiento entre el trigo (Triticum spp.) y el centeno (Secale cereale L.), comenzaron hace más de un siglo. El objetivo original era producir un tipo de cereal que complementara o superara al trigo y al centeno. Era un gran desafío, ya que el triticale tuvo problemas en sus inicios, debido a que por lo general las plantas eran muy altas, de madurez tardía, sensibles al fotoperíodo, y parcialmente estériles, con tendencia a producir semillas arrugadas. Estos problemas determinaron los primeros proyectos para mejorar el triticale (Varughese et al., 1987). Con el tiempo y mediante la mejora genética realizada por científicos de muchos países, se lograron adelantos significativos, resolviéndose en gran medida los problemas, lo cual ha permitido que actualmente se cultiven más de cuatro millones de hectáreas en el mundo (FAO, 2018).

Ammar et al. (2004) señalaron que si bien se siguen buscando los medios de elevar el rendimiento, mejorar la adaptación y su calidad industrial, en la actualidad se dedica mayor atención a desarrollar variedades que prosperen en ambientes agronómicamente subóptimos y que presenten resistencia a enfermedades. De acuerdo con Mayorga (1971), los estudios en triticales realizados en Chile comenzaron en 1970, cuando algunas líneas experimentales provenientes del CIMMYT (Centro Internacional de Mejoramiento de Maíz y Trigo) de México fueron introducidas y evaluadas por la Sociedad Nacional de Agricultura (SNA). Posteriormente, tanto la Pontificia Universidad Católica (Parodi y Nebreda, 1982) como el Instituto de Investigaciones Agropecuarias (INIA) (Hewstone, 1990; Mellado et al., 2008) y Semillas Baer (Baer, E. 2018. Semillas Baer. Comunicación personal), iniciaron trabajos de genética y agronomía del cereal, liberando variedades para el país, transformándose así en una alternativa comercial, que actualmente está inserta en la agricultura del centro sur y sur del país.

Es así como el Programa Nacional de Trigo del Instituto de Investigaciones Agropecuarias (INIA), como una forma de fortalecer este cultivo en el sur de Chile, ha desarrollado y mantenido trabajos en el área del mejoramiento genético, que han permitido en los últimos 35 años la liberación sostenida de variedades, entre las cuales se destaca Lonquimay INIA, Tolhuaca INIA, Peteroa INIA, Aguacero INIA y Faraón INIA, entre otras (Hewstone, 1986; 1990), lo que se traduce en que la superficie sembrada con triticale en Chile supere las 23.000 hectáreas (ODEPA, 2018), distribuyéndose entre las regiones del Biobío (4.069 has), La Araucanía (17.125 has), Los Ríos (925 has) y Los Lagos (2.000 has). Esto refleja la importancia que tiene el cultivo en la zona sur, y la necesidad de contar con variedades de mayores y mejores bondades que satisfagan las necesidades del agricultor y los requerimientos de la industria ganadera. Como una forma de cumplir con lo anterior, el INIA, a través de su Programa Nacional de Trigo con sede en INIA Carillanca (Región de La Araucanía), liberó recientemente una nueva variedad de triticale denominada Emperador INIA, para ser sembrada por los agricultores del sur de Chile.

\section{MATERIALES Y MÉTODO}

\section{Material vegetal}

La variedad Emperador INIA es producto de una cruza realizada en Alemania. Sus progenitores son la variedad de invierno Tulus y la línea experimental DNK0634, ambas pertenecientes a la empresa Nordsaat Saatzucht $\mathrm{GmbH}$, de Alemania. La variedad Tulus ha sido muy utilizada en programas de cruzas en INIA Carillanca, destacándose por su tipo y rendimiento; también ha sido una de las variedades más sembradas en varios países europeos en los últimos años, como Alemania, Polonia, Rusia, Hungría, Francia, entre otros (Ulrich, E., 2017. Nordsaat Saatzucht GMBH, Comunicación personal).

\section{Método}

La variedad Emperador INIA ha sido evaluada desde el año 2013 en un ensayo preliminar junto a otras variedades en la localidad de Vilcún (INIA Carillanca). Dado su tipo agronómico, características de grano y sanidad, fue posteriormente incorporado, en la temporada 2014/15, al ensayo estándar nacional que incluye 20 genotipos y variedades comerciales, siendo estudiado desde Chillán (36 $31^{\prime}$ S; $71^{\circ} 54^{\prime}$ O) hasta Purranque $\left(40^{\circ} 52^{\prime}\right.$ S; $\left.73^{\circ} 12^{\prime} \mathrm{O}\right)$, abarcando localidades geográficas con diferentes condiciones agroecológicas, que comprenden cuatro regiones del sur de Chile. La unidad experimental fue una parcela de $3 \mathrm{~m}$ de largo y 1,2 $\mathrm{m}$ de ancho ( 6 surcos a 0,2 m), cuya superficie fue de $3,6 \mathrm{~m}^{2}$; se usó un diseño de bloques completos al azar con cuatro repeticiones. El manejo agronómico fue óptimo, con el fin de detectar diferencias entre genotipos. La fertilización base fue entre 140 a $160 \mathrm{~kg} \mathrm{ha}^{-1} \mathrm{de}$ $\mathrm{N}, 100$ a $120 \mathrm{~kg} \mathrm{ha}^{-1}$ de $\mathrm{P}_{2} \mathrm{O}_{5^{\prime}}$ y entre 60 a $80 \mathrm{~kg} \mathrm{ha}^{-1}$ de $\mathrm{K}_{2} \mathrm{O}$, según la localidad y análisis de suelo.

Se realizó el correspondiente análisis paramétrico de la varianza y la prueba de Tukey para diferenciar promedios. Los caracteres de 
calidad del grano en las temporadas 2016 y 2017 se analizaron en el Laboratorio de Bromatología, CRI-Remehue, Osorno. Se determinaron: Materia seca (\%), Cenizas (\%), Proteína cruda $(\mathrm{N} \times 6,25)$, Digestibilidad materia seca (\%), Energía metabolizable (Mcal kg-1), Energía neta lactancia (\%) y Gluten húmedo (\%). La tolerancia al aluminio de la variedad Emperador INIA se estimó por medio de ensayo en suelo ácido usando la metodología de Peñaloza et al. (2009), considerando el peso fresco total de las raíces como variable de respuesta en lugar de su longitud. Diez plántulas de esta línea fueron sembradas en rizoboxes que contenían suelos con alto y bajo aluminio intercambiable. Las plantas de 'Emperador INIA' se cultivaron durante dos semanas, luego se aislaron y se pesaron las raíces.

\section{RESULTADOS Y DISCUSIÓN}

\section{Descripción morfológica de la planta}

La variedad Emperador INIA es un triticale de hábito de crecimiento invernal, lento al estado juvenil temprano, de porte muy rastrero, terminando con una abundante y vigorosa macolla, que cubre muy bien el suelo. Sus hojas son de color verde pálido y la hoja bandera semierecta. La espiga es de color rojo, semicurvada a curvada, de forma paralela, densidad alta, compacta y con barbas. El grano es de forma alargada, tamaño mediano, color rojo pálido, pudiendo presentar ocasionalmente ciertos cambios de pigmentación en el endosperma, con cepillo de largo mediano y surco central marcado. El peso promedio de 1000 granos es de 55,0 g, con un rango de 52,0 a 58,0 $\mathrm{g}$, dependiendo de la época de siembra y de la localidad. La altura de planta adulta varía entre 110 y $120 \mathrm{~cm}$, con un promedio de $115 \mathrm{~cm}$, siendo considerado un triticale de altura mediana, un poco más alto que la variedad Faraón INIA. Tiene tallo hueco, de mediana longitud, con moderada resistencia a la tendedura, por lo cual, bajo condiciones de alta productividad se recomienda el uso de regulador de crecimiento. Su período vegetativo, desde siembra a espigadura en Vilcún,

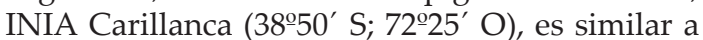
la variedad Faraón INIA. Cuando se siembra el 15 de mayo en INIA Carillanca, espiga entre el 09 y 12 de noviembre (180 días aproximadamente de siembra a espigadura). En la localidad de Purranque ( $\left.40^{\circ} 52^{\prime} \mathrm{S}, 73^{\circ} 12^{\prime} \mathrm{O}\right)$, Región de Los Ríos, sembrado el 20 de mayo, espiga alrededor del 20 al 23 de noviembre (183 días aproximadamente de siembra a espigadura), en un período que reúne entre 720 a 780 grados días, base $5^{\circ} \mathrm{C}$ en Vilcún, y entre 757 y 854 grados días, base $5^{\circ} \mathrm{C}$ en Purranque. Su tipo agronómico es similar a Faraón INIA, adaptado a las condiciones del sur, en ciclo de desarrollo, altura de planta y tolerancia a la tendedura.

\section{Características fitopatológicas}

Este cultivar es un triticale que ha presentado moderada susceptibilidad al polvillo estriado (Puccinia striiformis West. f. sp. tritici Erikss.) y resistencia al polvillo colorado de la hoja (Puccinia triticina f. sp. tritici Erikss. y Henn). Tiene moderada resistencia a septoriosis (Mycosphaerella graminicola (Fuckel) Sand., y es resistente a oidio (Blumeria graminis D.C. f. sp. tritici Marchal). Su comportamiento fitosanitario respecto a la roya amarilla es muy superior a la variedad Faraón INIA, que en la temporada 2018/19 mostró una fuerte sensibilidad a esta enfermedad. Sin perjuicio de lo anterior y debido al impacto que tuvo esta enfermedad en los triticales en general, se recomienda considerar una aplicación preventiva de fungicida.

\section{Rendimiento de grano}

El rendimiento de Emperador INIA se compara con los de Faraón INIA, dado que este es un cultivar de alto rendimiento. En todos los ensayos Emperador INIA superó a Faraón INIA. Su promedio, en las tres localidades y para los años de evaluación (Tabla 1), fue un $19,0 \%$ superior al testigo (15,0 versus $\left.12,6 \mathrm{t} \mathrm{ha}^{-1}\right)$ siendo equivalente a $2,4 \mathrm{t} \mathrm{ha}^{-1}$ más a favor de esta nueva variedad. La diferencia más notable entre estas dos variedades se presentó en la localidad de Purranque $\left(40^{\circ} 52^{\prime}\right.$ S; $73^{\circ} 12^{\prime} \mathrm{O}$ ), donde rindió un $28,7 \%$ más. Los rendimientos observados en Emperador INIA por sobre el testigo, confirman el alto potencial productivo que tiene y su adaptabilidad a las diferentes características ambientales del centro sur y sur de Chile. Se debe destacar que en la zona de Purranque $\left(40^{\circ} 52^{\prime}\right.$ S; $\left.73^{\circ} 12^{\prime} \mathrm{O}\right)$, Emperador INIA superó los $21,2 \mathrm{t} \mathrm{ha}^{-1}$, cifra que no ha sido alcanzada por ningún genotipo de triticale, $\mathrm{y}$ observada solo en la nueva variedad de trigo Kiron INIA durante la temporada 2014/15 en la misma localidad.

\section{Calidad}

Desde el punto de vista de calidad de grano (Tabla 2), el cultivar Emperador INIA es un triticale de peso del hectolitro superior a Faraón INIA y de textura de grano blanda. Presenta un porcentaje de proteína cruda superior al $11,0 \%$, un buen nivel de materia seca con alta digestibilidad, buen valor de energía metabolizable, siendo similar a Faraón INIA en todas las evaluaciones realizadas, excepto en proteínas, donde ha mostrado una tendencia a tener un valor superior. Se debe considerar que Faraón INIA es un triticale que reúne características de calidad 
Tabla 1. Rendimiento promedio de grano $\left(\mathrm{t} \mathrm{ha}^{-1}\right)$ de triticale variedad Emperador INIA comparado con Faraón INIA, en tres localidades del sur de Chile, durante las temporadas 2014/15 al 2016/17.

Table 1. Mean grain yield $\left(\mathrm{t} \mathrm{ha}^{-1}\right)$ of triticale $\mathrm{cv}$. Emperador INIA compared to Faraón INIA in three locations of south Chile during the seasons 2014/15 to 2016/17.

\begin{tabular}{|c|c|c|c|c|}
\hline Localidad & Temporada & $\begin{array}{c}\text { Rendimiento } \\
\text { Emperador } \\
\text { INIA* }^{*}\end{array}$ & $\begin{array}{l}\text { Rendimiento } \\
\text { Faraón INIA* }\end{array}$ & $\begin{array}{l}\text { CV } \\
(\%)\end{array}$ \\
\hline & & ------------' & רa-1--------- & \\
\hline \multirow[t]{3}{*}{ Chillán } & $2014 / 2015$ & $13,4 \mathrm{a}$ & $12,4 \mathrm{~b}$ & 11,69 \\
\hline & $2015 / 2016$ & $16,8 \mathrm{a}$ & $16,5 \mathrm{a}$ & 14,60 \\
\hline & $2016 / 2017$ & $14,9 \mathrm{a}$ & $13,3 \mathrm{~b}$ & 11,36 \\
\hline Promedio & & 15,0 & 14,1 & \\
\hline \multirow[t]{3}{*}{ Vilcún } & $2014 / 2015$ & $12,2 \mathrm{a}$ & $12,0 \mathrm{a}$ & 12,04 \\
\hline & $2015 / 2016$ & $11,3 \mathrm{a}$ & $10,4 \mathrm{a}$ & 11,73 \\
\hline & $2016 / 2017$ & $12,0 \mathrm{a}$ & $9,9 \mathrm{~b}$ & 6,82 \\
\hline Promedio & & 11,8 & 10,8 & \\
\hline \multirow[t]{3}{*}{ Purranque } & $2014 / 2015$ & $21,2 \mathrm{a}$ & $17,2 \mathrm{~b}$ & 8,12 \\
\hline & $2015 / 2016$ & 17,0 a & $14,0 \mathrm{~b}$ & 10,07 \\
\hline & $2016 / 2017$ & $16,2 \mathrm{a}$ & $13,4 \mathrm{~b}$ & 10,83 \\
\hline Promedio & & 18,1 & 12,9 & \\
\hline Promedio total & & 15,0 & 12,6 & \\
\hline
\end{tabular}

${ }^{*}$ Medias con la misma letra por temporada no difieren estadísticamente, Tukey $(\mathrm{P} \leq 0,05)$

para la alimentación de bovinos, porcinos y monogástricos en general (Jobet et al., 2010).

\section{Comportamiento a la acidez del suelo}

La estimación de la tolerancia al aluminio para este cultivar se infirió de las medias de peso fresco de raíces producidas en ensayos de corto plazo llevados a cabo en rizobox, empleando suelos de alto y bajo aluminio intercambiable. Se usó la variedad Faraón-INIA como testigo, cuya tolerancia al aluminio ha sido estudiada y demostrada en condiciones de campo.

Ambas variedades experimentaron una reducción esperada del peso fresco promedio de raíces por planta al ser cultivadas en suelo con alto aluminio intercambiable, respecto del peso obtenido en suelo con bajo aluminio intercambiable. La pérdida de peso fresco promedio de raíces fue de $72,9 \mathrm{mg}$ para Faraón (INIA, $\mathrm{p}<0,01)$ y de $35,7 \mathrm{mg}$ para Emperador INIA (no significativa, $p>0,05$ ), lo que representa pérdidas del 46 y $28 \%$, respectivamente. Estos resultados sugieren que Emperador INIA presentaría una mejor tolerancia al aluminio intercambiable respecto de Faraón INIA.

\section{Zona de cultivo y fecha de siembra}

Considerando su largo de ciclo, en zonas de primavera seca tiende a disminuir su productividad por ser un triticale de invierno, con requerimientos de frío y humedad en suelo, especialmente en el período de llenado de grano, por lo tanto su área de siembra debiera ajustarse a localidades de inviernos más largos y primaveras húmedas, semejante a la que tiene la región de Los Ríos y de Los Lagos en el sur de Chile. Se recomienda su siembra desde fines de abril hasta fines de junio, para la región del Maule al sur, siendo una variedad de gran potencial para la zona de los suelos trumaos y en sectores de precordillera.

\section{CONCLUSIÓN}

El cultivar Emperador INIA tiene características de potencial de rendimiento, sanidad, adaptabilidad y uso, que representa una alternativa para agricultores que buscan una variedad superior a Faraón INIA, especialmente en las regiones del sur de Chile, con suelos agrícolas con contenidos altos de aluminio fitotóxico y bajos $\mathrm{pH}$. La incorporación del cultivo de triticales que toleren mejor esta condición permite una respuesta en productividad y calidad de grano. 
Tabla 2. Características de calidad del triticale Emperador INIA comparada con Faraón INIA cultivados durante 2015/16 y 2016/17 en el sur de Chile.

Table 2. Quality characteristics of Emperador INIA triticale compared to Faraón INIA growing during 2015/16 and 2016/17 in the south of Chile.

\begin{tabular}{|c|c|c|c|c|c|c|}
\hline \multirow[t]{3}{*}{ Característica } & \multicolumn{6}{|c|}{ Variedad } \\
\hline & \multicolumn{3}{|c|}{ Emperador INIA* } & \multicolumn{3}{|c|}{ Faraón INIA* } \\
\hline & $2015 / 16$ & 2016/17 & Prom. & 2015/16 & 2016/17 & Prom. \\
\hline Materia seca, $\%^{1}$ & 87,1 & 87,2 & 87,2 & 87,1 & 87,2 & 87,2 \\
\hline Cenizas, \% & 1,4 & 1,7 & 1,6 & 1,3 & 1,5 & 1,4 \\
\hline Proteína cruda $(\mathrm{N} \times 6,25)^{2}$ & 10,8 & 11,8 & 11,3 & 10,0 & 11,4 & 10,7 \\
\hline Digestibilidad materia seca, $\%^{3}$ & 95,2 & 94,1 & 94,7 & 95,7 & 94,2 & 95,0 \\
\hline $\mathrm{D}, \%^{4}$ & 94,8 & 92,5 & 93,7 & 94,4 & 92,9 & 93,7 \\
\hline Energía metabolizable, Mcal kg-1 5 & 3,30 & 3,29 & 3,30 & 3,35 & 3,30 & 3,33 \\
\hline Energía neta lactancia, $\%{ }^{6}$ & 1,95 & 1,94 & 1,95 & 1,97 & 1,94 & 1,96 \\
\hline Gluten húmedo, $\%^{7}$ & - & 14,7 & 14,7 & - & 10,7 & 10,7 \\
\hline Peso hectolitro, $\mathrm{kg} \mathrm{hL}^{-18}$ & 76,9 & 76,8 & 76,9 & 76,1 & 75,2 & 75,7 \\
\hline
\end{tabular}

* Valores de las temporadas 2016 y 2017. Laboratorio de Bromatología, INIA CRI Remehue.

Superíndice. Se consideran buenos valores: ${ }^{1}$ Sobre $85 ;{ }^{2}$ Sobre $12 \% ;{ }^{3}$ Sobre $90 \% ;{ }^{4}$ Sobre $90 \% ;{ }^{5}$ Sobre $3,0 \% ;{ }^{6}$ Bajo $2 \%$; ${ }^{7}$ Sobre $26 \%$ buen valor para calidad panadera; ${ }^{8}$ Sobre $76 \mathrm{~kg} \mathrm{hL}^{-1}$

\section{LITERATURA CITADA}

Ammar, K., M. Mergoum, y S. Rajaram. 2004. The history and evolution of triticale. p. 1-9. In M. Mergoum and H. Gómez-MacPherson (eds.). Triticale improvement and production. Food and Agriculture Organization of the United Nations (FAO), Rome, Italy.

FAO. 2018. Estadísticas productivas. Disponible en htpp:/www.fao.org/faostat/ (Consulta 11 de octubre de 2018).

Hewstone, C. 1986. Avances en el mejoramiento de triticales para la zona sur de Chile. Simiente 56(3-4):139-145.

Hewstone, C. 1990. Triticale investigations in Chile. p. 586-592. In Proceeding of the Second International Triticale Symposium, Paso Fundo, Río Grande do Sul, Brasil. 1-5 October 1990. CIMMYT, EMBRAPA-CNPT, ITA, México.

Jobet, C., I. Matus, R. Campillo, O. Romero, R. Madariaga, y C. Alfaro. 2010. Faraón-INIA, un nuevo triticale de invierno $(\times$ Triticosecale Wittmack) para el Sur de Chile. Chilean Journal of Agricultural Research 70(4):670673.

Mayorga, I. 1971. Triticales en Chile. Simiente 41(3-4):52-53.

Mellado, M., I. Matus, y R. Madariaga. 2008. Antecedentes sobre el triticale en Chile y otros países. Boletín $\mathrm{N}^{\circ} 183.74$ p. Instituto de Investigaciones Agropecuarias INIA, Chillán, Chile.
ODEPA. 2018. Estadísticas productivas. Disponible en http:/www.odepa.gob.cl/ (Consulta 08 de octubre de 2018).

Peñaloza, E., J. Diaz, J. Zuñiga, P. Rathgeb, and A. Montenegro. 2009. Preliminary assessment of an in-soil and short-term method to screen for resistance to phytotoxic aluminum in wheat. Proceedings of VII Simposio de Recursos Genéticos para América Latina y el Caribe. 28-30 de octubre, Pucón, Chile. Volumen 1. p. 341-342. Instituto de Investigaciones Agropecuarias (INIA), Chile.

Parodi, P., e I. Nebreda. 1982. Seis años de investigación en triticale ( $\mathrm{x}$ Triticosecale Wittmack) en Chile. Ciencia e Investigación Agraria 9:15-25.

Varughese, G., T. Barker, y E. Saari. 1987. Triticale. 32 p. Centro Internacional de Maíz y Trigo (CIMMYT), México. 